\title{
SPRING MEETING OF THE SOCIETY AT CHICAGO.
}

THE thirty-third regular meeting of the Chicago Section of the American Mathematical Society was held at the University of Chicago on Friday and Saturday, April 10-11, 1914, it being the second regular meeting of the Society at Chicago. Seventy-four persons were in attendance upon the sessions, including the following forty-seven members of the Society: Professor R. P. Baker, Professor G. A. Bliss, Professor D. F. Campbell, Professor R. D. Carmichael, Dr. E. W. Chittenden, Mr. E. H. Clarke, Professor H. E. Cobb, Professor A. R. Crathorne, Professor D. R. Curtiss, Professor L. E. Dickson, Mr. C. R. Dines, Professor Arnold Dresden, Mr. M. G. Gaba, Professor E. R. Hedrick, Dr. T. H. Hildebrandt, Mr. L. A. Hopkins, Professor A. M. Kenyon, Professor W. C. Krathwohl, Dr. Charles Kuschke, Professor Kurt Laves, Professor A. C. Lunn, Professor W. D. MacMillan, Dr. H. F. MacNeish, Professor H. W. March, Mr. T. E. Mason, Professor G. A. Miller, Professor C. N. Moore, Professor E. H. Moore, Professor E. J. Moulton, Professor F. R. Moulton, Miss Ida M. Schottenfels, Mr. A. R. Schweitzer, Professor J. B. Shaw, Professor Mary E. Sinclair, Professor C. H. Sisam, Professor E. B. Skinner, Professor H. E. Slaught, Dr. E. B. Stouffer, Professor A. L. Underhill, Dr. G. E. Wahlin, Miss Mary E. Wells, Mr. C. W. Wester, Professor W. D. A. Westfall, Professor E. J. Wilczynski, Professor F. B. Wiley, Professor J. W. A. Young, Professor A. Ziwet.

Professor E. J. Wilczynski, Chairman of the Section and Vice-President of the Society, presided during the first three sessions and Professor G. A. Miller at the final session.

At the business meeting of the Section on Saturday morning it was voted that the program committee be instructed to set the date for the December meeting at such a time as not to conflict either with the annual meeting of the Society in New York or with the convocation of the American Association at Philadelphia, in order that members desiring to attend all three meetings may be free to do so.

General interest was manifested in the recent appeals of the Euler Commission for additional contributions and it was voted that the Section urge the desirability of further sup- 
porting in every practicable way the Euler publications. It may not be generally understood that only two of these volumes are appearing each year, so that the total annual cost of purchase is only about ten dollars.

In consequence of extended informal discussion begun at the dinner on Friday evening, it was voted that a committee of five be appointed by the chair to report to the Section at the December meeting, concerning possible recommendations to the Council of the Society on the question of the relation of the Society to the field now covered by the American Mathematical Monthly. The chair appointed as this committee Professors Alexander Ziwet, G. A. Miller, E. B. Van Vleck, E. R. Hedrick, and R. P. Baker.

The usual social gathering and dinner on Friday evening was attended by forty-two members and was pronounced by many to be the most enjoyable ever held by the Section.

The following papers were presented at this meeting:

(1) Mr. T. E. Mason: "On some properties of the elementary symmetric functions of the integers $1,2, \cdots, n-1$."

(2) Professor R. D. Carmichael and Mr. T. E. Mason: "Note on the roots of algebraic equations."

(3) Dr. G. E. WAHLin: "The logarithms of algebraic numbers in a domain $k(p, \mathfrak{a}) . "$

(4) Professor T. E. McKinney: "Some properties of $\lambda$ developments of quadratic irrationalities."

(5) Mr. Edward Kincher: "Group properties of the residue classes of certain Kronecker modular systems" (preliminary report).

(6) Professor G. A. Miller: "Note on multiply transitive solvable substitution groups.”

(7) Professor G. A. BuIss: "A note on symmetrical matrices."

(8) Mr. A. R. SchweItzen: "The aims and methods of mathematical research" (preliminary report).

(9) Professor A. B. Frizell: "On a certain series of nondenumerable sets."

(10) Dr. Henry Blumberg: "Certain general properties of functions."

(11) Dr. T. H. HildebrandT: "Note on monotoneity in continuous functions."

(12) Professors E. R. Hedrick and W. D. A. Westfall: "On functions defined implicitly in a region." 
(13) Mr. W. L. Hart: "Determination of the coefficients of a trigonometric series."

(14) Professor C. N. Moone: "On the relation between certain methods for summing divergent series."

(15) Professor A. M. KenYon: "The solution of a certain functional equation analogous to Fredholm's integral equation."

(16) Mr. A. R. Schweitzer: "On certain functional equations."

(17) Dr. E. B. Stouffer: "On ruled surfaces in space of five or less dimensions."

(18) Professor E. J. Wilczynskr: "On the metrical theory of plane curves."

(19) Professor W. D. MacMillan: "On certain analytic differential equations of the first order."

(20) Professor W. D. MacMillan: "A reduction of certain analytic differential equations to differential equations of an algebraic type. Second paper."

(21) Mr. T. E. MAson: "On properties of the solutions of linear $q$-difference equations with entire function coefficients."

(22) Professor F. R. Moulton: "On the stability of Jupiter's eighth satellite."

(23) Professor F. CAJori: "British discussions of Zeno's argumentation on motion."

(24) Professor A. D. Pitcher and Dr. E. W. Chittenden: "A theory of integration for classes which admit a development."

(25) Dr. E. W. Chittenden: "On the converse of the Heine-Borel theorem in domains for which 'limiting element' is defined."

(26) Professor R. P. BAKER: "On reflexion groups."

(27) Professor R. P. BAKer: "On Cayley diagrams."

(28) Professor L. E. Dickson: "On the twenty-eight bitangents to a quartic curve."

(29) Mr. A. R. Schweitzer: "Generalization of certain functional equations."

Mr. Hart was introduced by Professor F. R. Moulton and Mr. Kircher by Professor G. A. Miller. The papers of Professors McKinney, Frizell, Wilczynski, Dr. Blumberg, and the first and third papers of Mr. Schweitzer were read by title.

Abstracts of the papers follow, the numbers corresponding to those of the titles above. 
1. A well-known property of the elementary symmetric functions $A_{i}$ of the integers $1,2, \cdots, p-1$ is that each is divisible by $p$, when $p$ is prime, except $A_{p-1}$. Mr. Mason shows that those $A$ 's with odd subscripts are divisible by $p^{2}$ except $A_{1}$. This property enables him to prove the following congruence for $p$ a prime $>3$ and $n$ an integer:

$$
(n p) !-n !(p !)^{n} \equiv 0 \quad\left(\bmod p^{n+3}\right) .
$$

These properties of divisibility by $p$ of the elementary symmetric functions enable him to obtain properties concerned with the divisibility by $p$ of the sums of powers of the integers 1 , $\cdots, p-1$. These properties of the sums of powers furnish theoretical but impracticable tests for prime numbers and for determining the least prime factor of a given number.

2. This note by Professor Carmichael and Mr. Mason contains several theorems concerning the zeros of polynomials, of which the following is typical: Every equation of the form

$$
1+x+c_{s+1} x^{s+1}+c_{s+2} x^{s+2}+\cdots+c_{2^{2}} x^{2^{s}}=0 \quad(s \geqq 1),
$$

has at least one root not greater than 2 in absolute value, while special equations of this form may have all roots but one greater in absolute value than any preassigned $M$.

3. In his recent book Professor Hensel has developed a theory of logarithms of the rational $p$-adic numbers, and from this he has shown how all such numbers can be written in the form $p^{\alpha} \omega^{\beta} e^{\gamma}$.

Dr. Wahlin's paper is the result of the study of the same question with reference to the general algebraic domain $k(p, \mathfrak{a})$. He proves the existence of the logarithms of the numbers of $k(p, \mathfrak{a})$, and by considering certain group properties of the residues mod $p^{k}$, where $p^{k}$ is a certain power of the prime divisor $p$, the exponent being dependent upon certain properties of $k(p, a)$, he shows how we can have a representation of all numbers in $k(p, \mathfrak{a})$ similar to that obtained by Professor Hensel.

4. This note by Professor McKinney proves the periodic character of the $\lambda$-developments of quadratic irrationalities and determines an upper limit to the number of elements in the period. It shows that the $\lambda$-developments of the square 
roots of rational numbers are periodic with one and but one element preceding the period, and notes some further properties of these developments.

5. In this paper Mr. Kircher studies some of the group properties of the residue classes belonging to a Kronecker modular system of the form $\mathbf{M}=(\psi, \mathbf{M})$, where $\mathbf{M}$ is an ideal of the algebraic number domain $\Omega$ of degree $n$ and $\psi$ is a rational integral function of $x$ whose coefficients are algebraic integers of $\Omega$, the one belonging to the highest power of $x$ being equal to unity. A necessary and sufficient condition that a set of residue classes form a group with respect to the modular system $\mathbf{M}=\mathbf{M}^{\prime} \mathbf{M}^{\prime \prime}$ is found to be that all classes of the set must be contained in the modular system $\mathbf{M}^{\prime \prime}$ and must be relatively prime to the system $\mathbf{M}^{\prime}$. The second general result states that such a group belonging to $\mathbf{M}$ is equal to the direct product of the groups formed when the set of residue classes is taken with respect to each of the irreducible modular divisors of M. From these facts follow at once a number of generalizations, with respect to the modular system $\mathbf{M}$, of well-known theorems in number theory, such as those dealing with the value of the totient of $\mathbf{M}$ or the solution of a congruence of the first degree in one unknown, modulo $\mathbf{M}$.

6. Galois observed that a primitive substitution group cannot be solvable unless its degree is a power of some prime number. In fact, it is known that a solvable primitive substitution group is a subgroup of the holomorph of a regular abelian substitution group of order $p^{m}$ and of type $(1,1,1, \cdots)$. From this it results at once that such a group cannot be more than doubly transitive when $p>2$. When $p=2$ this group can be more than doubly transitive, since the symmetric group of degree 4 is solvable. Professor Miller proved that this symmetric group is the only solvable substitution group which is more than doubly transitive. The proof was partly based on the fact that a number of the form $2^{n}-1$ cannot be a power, higher than the first, of any prime number.

7. In a recent paper Dickson has given an elegant and elementary proof of the theorem that in a symmetric matrix of rank $r$ at least one principal minor of order $r$ is not zero. Wedderburn deduced the same result by proving, in a less 
elementary though very concise manner, that under the same circumstances the sum of the principal minors does not vanish. The purpose of the note by Professor Bliss is to exhibit a theorem, provable in elementary fashion, which includes both of the results just mentioned and which sets forth quite clearly some further properties of symmetric matrices.

8. In Mr. Schweitzer's paper the following four typical research acts in mathematics are considered: (1) generalization under discrimination of terms; (2) discrimination under generalization; (3) generalization under identification; (4) identification under generalization. It is important to note that these acts are not mutually exclusive, and that the generalization in question may be either intensive or extensive. Examples illustrating each case are given.

9. In a paper read at Chicago in December, 1913, Professor Frizell called attention to a non-denumerable well-ordered set obtained by methods different from those employed in forming Cantor's second ordinal class. The present paper exhibits an $\omega$-series of well-ordered sets which can be put into one-to-one correspondence neither with one another nor with the continuum. The process used here is more direct than that of the writer's December paper and, like it, is due to Cantor.

10. Sierpinski has shown that for any bounded function $f(x)$ the relation

$$
\omega \omega \omega f(x)=\omega \omega f(x)
$$

holds, where $\omega f(x)$ represents the oscillation of $f(x)$ at the point $x$. Let $\omega^{\prime} f(x)$ denote the oscillation of $f(x)$ when any set of the first category may be neglected (cf., for example, Baire, Acta Mathematica, volume 30 (1906), page 22); and let $\omega_{d} f(x)$ denote the oscillation of $f(x)$ when any denumerable set may be neglected. Dr. Blumberg proves the following relations:

$$
\omega^{\prime} \omega^{\prime} \omega^{\prime} f(x)=0, \quad \omega_{d} \omega_{d} \omega_{d} \omega_{d} f(x)=\omega_{d} \omega_{d} \omega_{d} f(x) .
$$

He shows by examples that the inequalities

$$
\begin{gathered}
\omega \omega \omega^{\prime} f(x) \neq \omega^{\prime} \omega^{\prime} f(x), \quad \omega_{d} \omega_{d} \omega_{d} f(x) \neq \omega_{d} \omega_{d} f(x), \\
\omega_{d} \omega_{d} \omega_{d} f(x) \neq 0
\end{gathered}
$$


are possible. The results may be extended to more general cases.

11. The paper by Dr. Hildebrandt considers some generalizations of the theorem of Dini: "A monotonic sequence of continuous functions which converges to a continuous function converges uniformly."

12. In this paper, Professors Hedrick and Westfall extend to any region the results obtained for the neighborhood of a point in the paper which they presented at the last summer meeting of the Society. It is shown that if the difference jacobian satisfies at every point of a region the condition prescribed in the previous paper at a fixed point, then continuous solutions exist along any path up to a frontier point; and that the inverse of a plane transformation, for a convex region, exists throughout that region, under conditions analogous to the preceding.

13. In connection with certain problems in applied mathematics there arise series of the type

$$
f(t)=\sum_{i=0}^{\infty} A_{i} \cos 2 \pi a_{i} t+B_{i} \sin 2 \pi a_{i} t \quad\left(a_{0}=0\right) .
$$

In particular, the tidal effects of the moon and sun on the earth can be expanded in this form. In Professor A. A. Michelson's recent experiment for determining the rigidity of the earth by tidal effects, it was necessary to consider the determination of the coefficients of this expansion.

In this paper Mr. Hart considers the problem of finding the coefficients of a series of the form (1) when the function $f(t)$ is given, for example by observations, and the quantities $a_{i}$ are given by theory. Under the hypothesis that $\Sigma_{i=0}^{i=\infty}\left|A_{i}\right|$ $+\left|B_{i}\right|$ converges, it is shown that

$$
\begin{aligned}
& A_{0}=\delta \prod_{k=\infty} \frac{1}{k} \int_{0}^{k / \delta} f(t) d t \quad(\delta>0, \text { chosen arbitrarily }), \\
& A_{i}=2 a_{i} \mathbf{L}_{k=\infty} \frac{1}{k} \int_{0}^{k / a_{i}} f(t) \cos 2 \pi a_{i} t d t \quad(i=1,2, \cdots), \\
& B_{i}=2 a_{i} \mathbf{L}_{k=\infty} \frac{1}{k} \int_{0}^{k / a_{i}} f(t) \sin 2 \pi a_{i} t d t \quad(i=1,2, \cdots) .
\end{aligned}
$$


The hypothesis of this proof is satisfied in the case of the tidal effects except possibly for the terms due to the perturbative action of the sun on the moon. The expressions for $A_{i}$ and $B_{i}$ usually converge rapidly unless there are some other terms with large coefficients for which the quantities $a_{j}$ are nearly equal to $m_{j} a_{i}$.

14. In a memoir of $1908^{*}$ de la Vallée Poussin introduced the following formula for the summation of a divergent series $\Sigma u_{n}$ :

$$
\lim _{n=\infty}\left[u_{0}+\sum_{k=1}^{k=n} \frac{n(n-1) \cdots(n-k+1)}{(n+1)(n+2) \cdots(n+k)} u_{k}\right] .
$$

He further showed that this method of summation is applicable for the purpose of summing not only the Fourier's series of a wide class of functions but also any of the successive term by term derivatives of these series, at any point where the derivative of the function exists. It follows then that this method will enable us to sum series that would not be summable by a Cesàro mean of given order. We are thus led to inquire if de la Vallée Poussin's formula is more general than any of Cesàro's means, i. e., if a series summable $(C r)$ for any $r \geqq 0$ will be summable by the above formula and to the same value. In Professor Moore's paper this problem is considered and the question is answered in the affirmative.

15. It is the object of this paper to show that the body of theorems and formulas which effect the complete solution of the Fredholm integral equation

$$
f(x)=u(x)-\lambda \int_{a}^{b} K(x, \xi) u(\xi) d \xi
$$

and which are based upon the properties of the definite integral apply to the analogous functional equation

$$
f(x)=u(x)-\lambda\left[\frac{\partial}{\partial \xi} K(x, \xi) u(\xi)\right]_{\xi=a}
$$

* "Sur l'approximation des fonctions d'une variable réelle et de leurs derivées par des polynômes et des suites limitées de Fourier," (Bulletin de la classe des Sciences de l'Académie Royale de Belgique, 1908, pp. 193-254). 
with no further change than the substitution throughout of the operator

$$
\left[\frac{\partial}{\partial \xi}(\quad)\right]_{\xi=a} \text { for the operator } \int_{a}^{b}(\quad) d \xi .
$$

This solution, therefore, presents an illustration of the general remark that the Fredholm theory does not in fact depend upon the special properties of the definite integral, but only upon its two general properties: (1) that it is linear, and (2) that it reduces a function of two variables to a function of one variable; and upon a certain convergence condition.

Professor Kenyon further shows that, for all kernels which admit continuous second partial derivatives in a certain region of the $x y$-plane containing the point $(a, a)$, the Fredholm determinant $D(\lambda)$ and its first and second minors are polynomials in $\lambda$ of degree not greater than $2,3,4$ respectively; consequently, the index of a characteristic constant can not in any case exceed two.

16. Mr. Schweitzer discusses the solution of the equations

$$
\begin{gathered}
\psi\{\theta(x)-\theta(y)\}=\{\psi \theta(x+y)\}^{c}\{\psi \theta(x-y)\}^{c}, \\
\psi_{1}\left\{\theta_{1}(x)+\theta_{1}(y)+k\right\}=\left\{\psi_{1} \theta_{1}(x+y)\right\}^{c_{1}}\left\{\psi_{1} \theta_{1}(x-y)\right\}^{c_{1}}
\end{gathered}
$$

where $k, c$ and $c_{1}$ are constants, $\psi(x)$ and $\psi_{1}(x)$ are given functions, and $\theta_{1}(x)$ and $\theta(x)$ are to be determined.

17. In a previous paper Dr. Stouffer has investigated the projective differential properties of a three-spread generated by a single infinity of planes in $S_{5}$ by means of a system of three linear homogeneous differential equations of the second order. In the present paper he uses the same system for the study of ruled surfaces in $S_{5}$. The ruled surface may be of the general type or it may lie entirely in an $S_{3}$ or in an $S_{4}$ or have three consecutive generators in the same $S_{4}$. Certain invariant conditions upon the coefficients of the system must be satisfied in each of the special cases. The only ruled surfaces excluded from the theory are those which are developable in the ordinary sense. Several sets of curves upon the surface and a number of different types of derivative surfaces are discussed. 
18. The metric theory of plane curves is a far richer field than would appear from the presentations of the subject which have become classic. The results of Abel Transon in 1841 have been almost completely ignored by the writers on this subject. The more recent important contributions of Cesàro are also practically unknown to the general mathematical public. The object of Professor Wilczynski's paper is a systematic unified treatment of the whole subject involving, besides certain new matters of detail, as its distinctly new features the introduction of the notions of projective differential geometry into the metric theory.

19. Consider a differential equation of the first order

$$
\frac{d x}{d t}=x^{n}+a_{1} x^{n+1}+a_{2} x^{n+2}+\cdots,
$$

the right member of which is a convergent power series in $x$ with constant coefficients, and in which $n$ is a positive or negative integer or zero. It is shown by Professor MacMillan that there exists a convergent series

$$
x=y+b_{2} y^{2}+b_{3} y^{3}+\cdots
$$

which, substituted in (1), reduces the differential equation to the form

$$
\frac{d y}{d t}=y^{n}+c_{n} y^{2 n-1},
$$

where $c_{n}=0$ if $n<2$, while in general $c_{n} \neq 0$ if $n \geqq 2$. If $n<2$ the integration of (2) is immediate. If $n \geqq 2$ and $c_{n} \neq 0$, the substitution

$$
c_{n} y^{n-1}=\frac{1}{W-1}, \quad 1-\frac{(n-1)}{c_{n}}\left(t-t_{0}\right)=\tau
$$

reduces the differential equation to the simple form

$$
\frac{d W}{d \tau}=\frac{W}{W-1},
$$

which is independent of $n$. The solution $W-\log W=\tau$ defines $W$ as a function of $\tau$. Writing it $W=L(\tau)$ and taking $L^{\prime}=d L / d t$, the solution of (2) becomes

$$
y=\left(\frac{L^{\prime}-1}{c_{n}}\right)^{1 / n-1} .
$$


The functions $L$ and $L^{\prime}$, which thus play an important rôle in the theory of the solutions of (1), are studied in detail.

20. Suppose that we have given a set of differential equations

$$
\frac{d x_{i}}{d t}=X_{i}\left(x_{1}, \cdots, x_{n}\right) \quad(i=1, \cdots, n)
$$

and that $X_{i}(0, \cdots, 0)=0$. Suppose further that the functions $X_{i}$ are analytic in $x_{1}, \cdots, x_{n}$ and regular at the point $x_{1}=\cdots=x_{n}=0$. At the last meeting in Chicago Professor MacMillan showed that if the characteristic equation formed from the determinant $\left|\partial X_{i} / \partial x_{j}\right|$ at the origin has only simple roots, then a convergent substitution

$$
x_{i}=P_{i}\left(y_{1}, \cdots, y_{n}\right)
$$

exists, whose jacobian $\left|\partial P_{i} / \partial y_{j}\right|$ does not vanish at the origin, and such that the resulting differential equations assume the simple form

$$
\frac{d y_{i}}{d t}=\alpha_{i} y_{i},
$$

where the constants $\alpha_{i}$ are the roots of the characteristic equation. This was done on the assumption that the constants $\alpha_{i}$ considered as points in the complex plane all lie on one side of a straight line through the origin, and further that there exists no set of integers $j_{1}, \cdots, j_{n}$ for which $j_{1}+j_{2}+\cdots$ $+j_{n} \geqq 2$ and such that $j_{1} \alpha_{1}+j_{2} \alpha_{2}+\cdots+j_{n} \alpha_{n}-\alpha_{i}=0$.

In the present paper the discussion is extended to the case where the characteristic equation has multiple roots of any order of multiplicity, and it is shown that if the last two conditions just stated are satisfied, the differential equations reduce to the form

$$
\frac{d y_{i}}{d t}=\alpha_{i} y_{i}+\sum_{j=1}^{i-1} a_{i j} y_{j},
$$

where the $\alpha_{i}$ 's are the roots of the characteristic equation and where the $a_{i j}$ 's have the value 0 or 1 according to the nature of the multiplicity of the roots.

If the roots $\alpha_{i}$ are simple roots and all lie on one side of a straight line and the condition $j_{1} \alpha_{1}+j_{2} \alpha_{2}+\cdots j_{n} \alpha_{n}-\alpha_{i}=0$ is satisfied by certain sets of integers for which $\Sigma_{k=1}^{k=n} j_{k} \geqq 2$, 
then there exist only a finite number of such sets and the differential equations reduce to the form

$$
\frac{d y_{i}}{d t}=\alpha_{i} y_{i}+\Sigma b_{j_{1} \ldots j_{n}}^{(i)} y_{1}^{j_{1}} \cdots y_{n}^{j_{n}} \quad(i=1, \cdots, n)
$$

where the summation is extended over all sets for which the condition $j_{1} \alpha_{1}+\cdots+j_{n} \alpha_{n}-\alpha_{i}=0$ is satisfied. To each of these extra terms in the differential equation there corresponds an arbitrary constant in the substitution.

21. In this paper Mr. Mason studies the existence and properties of entire functions defined by linear $q$-difference equations, homogeneous and non-homogeneous, with coefficients which are entire functions. Entire function solutions are shown to exist in case there are formal expansions which satisfy the equation. In certain other cases solutions exist which consist of an entire function multiplied by a function which has a singularity at the point zero.

If the coefficients are of finite class, the entire functions defined by such equations are of "ordre apparent," in the Borel sense, not greater than the greatest among the "ordres apparents " of the coefficients. When the coefficients are of infinite, but not transfinite, class the solutions are at most of infinite, but not transfinite, class.

22. If the eccentricity of the orbit of a planet is neglected, there is a well-known sufficient condition that a satellite revolving around it shall be permanently under the gravitational control of its primary. This condition, which was first used by Hill in the lunar theory, depends upon the character of the surfaces of zero relative velocity associated with Jacobi's integral. All the known satellites in the solar system fulfill this condition except JVIII; consequently its stability or instability must be established by other methods. Professor Moulton treats the question by first finding a periodic orbit having the same period (the motion is retrograde), and then discussing the stability of the associated periodic orbit according to the definition adopted by Poincaré. The discussion involves the treatment of certain linear differential equations having periodic coefficients, and it turns out that the orbit is stable. 
23. Professor Cajori's paper gives a critical account of the views on the theory of limits, infinite divisibility, and Zeno's arguments, held by Roger Bacon, Duns Scotus, Hobbes, Newton, Berkeley, Jurin, Robins, Hutton, Coleridge, De Quincey, De Morgan, Spencer, Mill, Hobson, Bertrand Russell, G. H. Hardy, and others.

24. In terms of a postulated development $\Delta=\left(\left(\mathfrak{B}^{m l}\right)\right)$ of a class $\mathfrak{B}$ and an associated system $\left(\left(d^{m l}\right)\right)$ of positive real numbers Professor Pitcher and Dr. Chittenden define a developmental integral, integral $(\Delta)$, of real valued functions on $\mathfrak{B}$. The theory is first treated in general, and the integral $(\Delta)$ is found to be an example of the type of linear operator $J$ used in the general integral equation theory of Professor E. H. Moore. Later, restrictions are placed upon the system which lead to a theory of measure for the class $\mathfrak{B}$, generalizing the theory of Lebesgue. A great majority of the existent types of definite integrals, both proper and improper, are included under this generalization.

25. Dr. Chittenden shows that in a domain admitting a relation "limiting element" between classes and single elements, which satisfies the postulates of F. Riesz,* every class $\mathfrak{Q}$, possessing the property characteristic of the Heine-Borel theorem, has the further property that every infinite subclass of $\mathfrak{Q}$ has a limiting element in $\mathfrak{Q}$. This result is applied to cases where "limiting element" and "interior to a class" are defined in terms of "limit of a sequence." This relation is taken in the general sense of T. H. Hildebrandt. $\dagger$ As a special result we have the following generalization of results of M. Fréchet and Hildebrandt: if the limit relation satisfies the Fréchet conditions, then we may modify the conclusion above to read, $\mathfrak{Q}$ is extremal, that is, compact and closed.

26. In this paper, Professor Baker shows that the symmetric group $G_{k}$ can be represented as a reflexion group in $R_{k-1}$. The matrix $\left|\alpha_{i k}\right|$ with the elements

$$
\alpha_{i i}=\frac{\sqrt{i+1}}{\sqrt{2 i}}, \quad \alpha_{i, i-1}=\frac{\sqrt{i-1}}{\sqrt{2 i}} \quad(i=1,2, \cdots, k-1),
$$

* "Stetigkeitsbegriff und abstracte Mengenlehre," Atti del IV ${ }^{\circ}$ Congresso Internazionale di Matematichi, Roma, 1908 , vol. 2, pp. 18-24.

$\dagger$ "A contribution to the foundations of Fréchet's calcul fonctionnel, Amer. Journal, vol. 34, p. 241. 
and all other elements zero, represents by its rows a set of $k-1$ axes. Each axis makes an angle of $120^{\circ}$ with its nighbors and is perpendicular to the others.

Reflexions in the flat $R_{k-2}$ perpendicular to these axes have the properties $(a)$ each is of period 2, (b) the product of two neighbors is of period 3, (c) the product of other pairs is of period 2.

These relations determine $G_{k}$ by Moore's theorem (Proceedings of the London Mathematical Society, volume 28). The $R_{k-2}$ 's divide the space into $k$ sectors which represent the elements of the group. By choice of a fundamental region consisting of a connected set of these sectors the elements of any subgroup can be represented.

By adding a $k$ th row to the matrix

$$
\begin{aligned}
\alpha_{k i}=-\frac{1}{\sqrt{2 i(i+1)}}, \quad \alpha_{k, k-1}= & -\frac{\sqrt{k}}{\sqrt{2(k-1)}} \\
& (i=1,2, \cdots, k-2),
\end{aligned}
$$

the reflexions in the $k R_{k-2}$ 's are in correspondence with the substitutions (01), (12), (23), $\cdots,(k-1,0)$. The $R_{k-2}$ 's not having the symbol $l$ determine a line containing the $l$ th vertex of the regular figure (simplex) with $k$ vertices in $R_{k-1}$.

27. All Cayley diagrams (color groups) of the plane have been determined by Maschke (American Journal, volume 18). The second paper of Professor Baker is concerned with the problem of finding all the Cayley diagrams representing a given group.

One such exists, namely the one obtained by constructing the Dyck figure, taking the polar figure, and suppressing the line representing the redundant generator. With such a figure, or any Cayley diagram otherwise obtained, the construction of all the others is reducible to a purely topological problem. In the case where three lines meet at each point the problem is simply stated: Place a mark on any arbitrary set of the lines and draw lines within each polygon crossing at the mark. The result is a symbol of a Cayley diagram in the most general sense. Namely, the lines which are necessarily closed represent the new polygons.

The number is in general so large that enumeration is impossible without further restrictions. 
Usual restrictions are (1) the polygons shall not self-touch, (2) the resulting figure shall be two-sided. For the octahedron group, as generated by three elements of order two, the unrestricted number is $2^{36}$ and no solution has more than 144 conjugate representations. With (1) alone the number is still probably too large for detailed enumeration. With (1) and (2) the number of distinct types is 61 . This and the number 73 of types of 'routes' for the group were determined by the use of sieves.

The abstract formulation of the topological problem is:

Given a complex $C_{2}$ by means of its matrices $X_{01}, X_{02}$; how many complexes are there having $X_{01}$ and a different $X_{02}(a)$ in general, (b) with restrictions (1) and (2), or both?

28. If $a$ and $b$ are any two bitangents to a general quartic curve, it is well known that there are just five new pairs of bitangents $c, d ; \cdots ; k, l$ such that the eight points of contact of $a, b, c, d$ are on a conic, $\cdots$, those of $a, b, k, l$ on a conic. Such a set of six pairs of bitangents $a b, c d, e f, g h, i j, k l$ is called a Steiner set (or group). The same set is uniquely determined by any one of its six pairs. The set determined by $a$ and $c$ contains the pair $b, d$, but no further bitangent in common with the first set. Professor Dickson considers the abstract problem to distribute 28 symbols into sets of six pairs such that

(i) Every pair occurs in one and but one set;

(ii) If $A B, C D$ are two pairs in a set, then $A C, B D$ are pairs in a set;

(iii) Two sets $A B, C D, \cdots$ and $A C, B D, \cdots$ have only four symbols in common.

In view of the theorems quoted, there is such a distribution of the 28 bitangents. It is shown that, in the abstract problem, any distribution can be derived from one distribution by a mere permutation of the symbols. The 28 symbols used in the proof are $12,13, \cdots, 78$, where $i j$ and $j i$ are identified. A distribution, from which every distribution can be derived by permutations of the 28 symbols, is composed of 63 sets falling into two types:

$a b c d, a c b d, a d b c$, ef $g h, e g f h, e h f g ;$

ag ah, bg bh, cg ch, dgdh, egeh, fg fh;

where $a, \cdots, h$ is any permutation of $1, \cdots, 8$.

This notation of Hesse therefore presents a lack of sym- 
metry as regards the distribution of the 28 bitangents into Steiner sets, whereas there is no geometrical distinction between sets of different types.

Perfect symmetry results if we employ the 28 symbols

$$
\left(x_{1}, y_{1}, x_{2}, y_{2}, x_{3}, y_{3}\right), x_{1} y_{1}+x_{2} y_{2}+x_{3} y_{3} \equiv 1(\bmod 2) \text {, }
$$

where each "element" $x_{i}$ and $y_{i}$ is 0 or 1 , corresponding to the theta functions for $p=3$ of odd characteristics. It is easy to prove that, if $A$ and $B$ are any two of these symbols, there exist exactly five pairs of symbols $C_{j}, D_{j}(j=1, \cdots, 5)$, distinct from each other and from $A$ and $B$, such that the six sums of corresponding elements (for example, their four elements $x_{1}$ ) of the four symbols $A, B, C_{j}, D_{j}$ are even.

It follows that the sums of corresponding elements of $C_{i}, D_{i}$, $C_{j}, D_{j}$ are even. Hence the set of six pairs determined by $A$ and $B$ is identical with the set of six pairs determined by $C_{i}, D_{i}$.

Under this law of separation into sets of six pairs, properties (i) and (ii) therefore hold, likewise (iii), as easily verified. It follows from our abstract theorem that the 28 bitangents can be designated by our new symbols in such a way that the eight points of contact of four bitangents are on a conic if and only if the six sums of corresponding elements of their four symbols are all even. We thus have an elementary proof of the last theorem, previously established by Riemann, Weber, and Clebsch by means of the theory of abelian functions. References are given in Annals of Mathematics, 1905, pages 141150 , where the theorem is shown to lead in a simple manner to the result that exactly $4,8,16$ or 28 of the bitangents are real.

29. Mr. Schweitzer investigates the following generalizations of functional equations previously discussed by him:

$$
\begin{aligned}
& \psi\{\theta(x) \phi(y)-\theta(y) \phi(x)\}=\{\psi F(x+y)\}^{l} \cdot\{\psi F(x-y)\}^{m}, \\
& \psi_{1}\left\{\theta_{1}(x) \theta_{1}(y)-\phi_{1}(y) \phi_{1}(x)\right\}=\left\{\psi_{1} F_{1}(x+y)\right\}^{l} \cdot\left\{\psi_{1} F_{1}(x-y)\right\}^{m} .
\end{aligned}
$$

In these equations $l, m, l_{1}, m_{1}$ denote constants.

H. E. Slaught, Secretary of the Chicago Section. 